\title{
Correction to: Are Clerks Proficient in the Basic Sciences? Assessment of Third-Year Medical Students' Basic Science Knowledge Prior to and at the Completion of Core Clerkship Rotations
}

\author{
Madeleine E. Norris ${ }^{1} \cdot$ Mark A. Cachia $^{2} \cdot$ Marjorie I. Johnson ${ }^{1} \cdot$ Charys M. Martin ${ }^{1} \cdot$ Kem A. Rogers ${ }^{1}$
}

Published online: 2 November 2021

(c) International Association of Medical Science Educators 2021

Correction to: The AAPS Journal (2021) 23:48

https://doi.org/10.1007/s40670-021-01249-3

The article has been updated to add funding information Schulich School of Medicine \& Dentistry's Summer Research Training Program (University of Western Ontario) and update the affiliations for Mark A. Cachia, Schulich School of Medicine \& Dentistry, University of Western Ontario, London, Ontario, Canada.

Publisher's Note Springer Nature remains neutral with regard to jurisdictional claims in published maps and institutional affiliations.

The original article can be found online at https://doi.org/10.1007/ s40670-021-01249-3.

Charys M. Martin

Charys.Martin@schulich.uwo.ca

Madeleine E. Norris

mnorris6@uwo.ca

Mark A. Cachia

mcachia2020@meds.uwo.ca

Marjorie I. Johnson

Marjorie.Johnson@schulich.uwo.ca

Kem A. Rogers

Kem.Rogers@schulich.uwo.ca

1 Department of Anatomy and Cell Biology, Schulich School of Medicine \& Dentistry, The University of Western Ontario, London, ON, Canada

2 Schulich School of Medicine \& Dentistry, University of Western Ontario, London, Ontario, Canada 